EESTI NSV TEADUSTE AKADEEMIA TOIMETISED. X KÖIDE FOUSIKALIS MATEMAATILISTE JA TEHNILISTE TEADUSTE SEERIA. 1961, NR. I

ИЗВЕСТИЯ АКАДЕМИИ НАУК ЭСТОНСКОИ ССР. ТОМ Х СЕРИЯ ФИЗИКО-МАТЕМАТИЧЕСКИХ И ТЕХНИЧЕСКИХ НАУК. 1961, N2 1

\title{
О ВАРИАЦИОННОЙ ЗАДАЧЕ КАСТИЛЬЯНО ДИНАМИКИ НЕЛИНЕИНОЙ ТЕОРИИ УПРУГОСТИ
}

\author{
Л. АинолА,
}

кандидат физико-математических наук

Имеется ряд попыток формулировки вариационной задачи Кастильяно в статике нелинейной теории упругости $\left[{ }^{1-5}\right]$. В этих работах либо варьируемые напряжения должны удовлетворять системе дифференциальных уравнений, содержащей неварьируемые перемещения, либо соответствующий функционал фигурирует в неявном виде. Для динамики линейной теории упругости вариационная задача Кастильяно дана в работах $[6,7]$. В настоящей статье общим методом преобразования вариационных задач ${ }^{[8]}$ из вариационной задачи Гамильтона выводится вариационная задача Кастильяно динамики нелинейной теории упругости.

\section{1. Исходная вариационная задача}

По принципу Гамильтона действительное движение упругой системы между двумя заданными конфигурациями отличается от кинетических возможных движений, совершаемых между теми же конфигурациями и в тот же самый промежуток времени, тем, что для действительного движения

$$
\delta \int_{i_{0}}^{t_{1}}(K-W) d t+\int_{i_{0}}^{t_{1}} \delta U d t=0 .
$$

Здесь $K$ - кинетическая энергия упругого тела, $W$ - потенциальная энергия упругого тела и $\delta U$ - работа, совершаемая внешними силами при вариациях перемещений. $K, W$ и $\delta U$ можно выразить в виде

$$
\begin{aligned}
& W=\int_{\dot{V}} F_{\mathrm{Q}}^{*} d V=\int_{\dot{V}} F_{\mathrm{Q}} d V, \\
& K=\frac{1}{2} \int_{\dot{V}}\left(\frac{\partial \bar{u}}{\partial t}\right)^{2} \varrho d \vec{V}=\frac{1}{2} \int_{V}\left(\frac{\partial u}{\partial t}\right)^{2} \varrho d V
\end{aligned}
$$

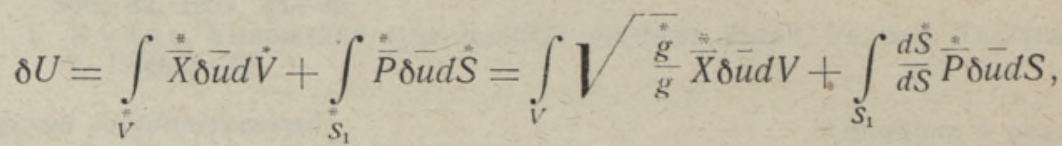

где $F$ - плотность потенциильной энергии деформированного упругого 
тела, отнесенная к единице массы; $\varrho$ и $Q-$ плотность деформированного и недеформированного упругого тела; $\bar{u}$ - вектор перемещения; $\overline{\bar{X}}$ и $\bar{P}$ - соответственно векторы объемных сил и нагрузки, отнесенных соответственно к единице объема и поверхности деформированного тела; $\stackrel{*}{V}, V$ и $\stackrel{\ddot{S}}{S}_{1}, S_{1}$ - объем и та часть поверхности, где задана внешняя нагрузка деформированного и недеформированного тела.

Если обозначить

$$
\begin{aligned}
& \bar{X}=\sqrt{\frac{\dot{*}}{g}} \frac{*}{X}, \\
& \bar{P}=\frac{d \dot{S}}{d S} \frac{*}{P}
\end{aligned}
$$

и предположить, что силы $\bar{X}$ и $\bar{P}$ не зависят от перемещений, то

$$
U=\int_{V} \bar{X} \bar{u} d V+\int_{S_{1}} \bar{P} \bar{u} d S .
$$

Теперь можно (1.1) переписать в виде

$$
\delta\left\{\int_{t_{0}}^{t_{1}} \int_{V}\left[\frac{1}{2} \varrho\left(\frac{\partial \bar{u}}{\partial t}\right)^{2}-\varrho F+\bar{X} \bar{u}\right] d V d t+\int_{t_{0}}^{t_{1}} \int_{S_{1}} \bar{P} \bar{u} d S d t\right\}=0 .
$$

Отметим, что плотность потенциальной энергии $F$ является функцией от компонентов тензора деформаций $\varepsilon_{i k}$. Последние выражаются через компоненты кинетического тензора

следующим образом:

$$
e_{i k}=\nabla_{i} u_{k}
$$

$$
\varepsilon_{i k}=\frac{1}{2}\left(e_{i k}+e_{k i}+g^{\alpha \beta} e_{i \alpha} e_{k \beta}\right) .
$$

В случае физически линейных задач

$$
\mathrm{Q} F\left(\varepsilon_{i k}\right)=\frac{1}{2} E^{\alpha \beta \gamma \hat{\jmath}} \varepsilon_{\alpha \beta} \varepsilon_{\gamma \delta},
$$

где $E^{i k j l}$ - тензор упругости.

Далее, обозначим компоненты вектора скорости через $v_{i}$, т. е.

$$
\frac{\partial u_{i}}{\partial t}=v_{i}
$$

и считаем, что в моменты $t_{0}$ и $t_{1}$ тело претерпевает перемешения, даваемые соответственно векторами $S_{2}$ поверхности тела даны геометрические граничные условия

$$
u_{i}-u_{i}^{\prime}=0 \text {. }
$$


Теперь из (1.8) получаем следующую исходную вариаци́онную задачу:

найти стационарное значение функционала

$$
\int_{t_{0}}^{t_{1}} \int_{V}\left[\frac{1}{2} \varrho v^{\alpha} v_{\alpha}-\varrho F\left(\varepsilon_{i k}\right)+X^{\alpha} u_{\alpha}\right] d V d t+\int_{t_{0}}^{t_{1}} \int_{S_{1}} P^{\alpha} u_{\alpha} d S d t
$$

при условиях

$$
\begin{aligned}
& u_{i}\left(x, t_{0}\right)-w_{i}=0, \\
& u_{i}\left(x, t_{1}\right)-w_{i}^{\prime}=0
\end{aligned}
$$

и граничных условиях (1.13) на $S_{2}$.

Как известно, в статике линейной теории упругости вариационная задача Кастильяно получается из вариационной задачи Лагранжа при помощи преобразования, аналогичного преобразованию Фридрихса. Наша цель - найти соответствующую преобразованную вариационную задачу для только что сформулированной вариационной задачи Гамильтона.

\section{2. Общая вариационная задача}

Чтобы получить вариационную задачу, которая имеет своим стационарным условием кроме уравнений равновесия еще геометрические соотношения и соотношения упругости, интерпретируем исходную вариационную задачу так, что кинетические величины $\varepsilon_{i k}, e_{i k}$ и $v_{i}$ фигурируют в ней как независимые функциональные аргументы. Конечно, эти величины должны удовлетворять дополнительным условиям (1.9), $(1.10)$ и (1.12). Если теперь при помощи правила множителей Лагранжа освободиться от дополнительных условнй (1.9), (1.10), (1.12), а также от граничных и начальных условий (1.13), (1.15), (1.16), то получается следующая общая вариационная задача:

найти стационарное значение функционала

$$
\begin{gathered}
\int_{t_{0}}^{t_{1}} \int_{V}\left\{\frac{1}{2} \varrho v^{\alpha} v_{\alpha}-\varrho F\left(\varepsilon_{i k}\right)+X^{\alpha} u_{\alpha}+\tau^{\alpha \beta}\left[\varepsilon_{\alpha \beta}-\frac{1}{2}\left(e_{\alpha \beta}+e_{\beta \alpha}+g \bar{\gamma}^{\bar{z}} e_{\alpha \gamma} e_{\beta \sigma}\right)\right]+\right. \\
\left.+\sigma^{\alpha \beta}\left(e_{\alpha \beta}-\nabla_{\alpha} u_{\beta}\right)-\varrho \lambda^{\alpha}\left(v_{\alpha}-\frac{\partial u_{\alpha}}{\partial t}\right)\right\} d V d t+\int_{t_{0}}^{t_{1}} \int_{S_{1}} P^{\alpha} u_{\alpha} d S d t- \\
+\int_{t_{0}}^{t_{1}} \int_{S_{2}} \vartheta^{\alpha}\left(u_{\alpha}^{\prime}-u_{\alpha}\right) d S d t+\int_{V}\left\{\varrho \mu^{\alpha}\left[w_{\alpha}^{\prime}-u_{\alpha}\left(x, t_{1}\right)\right]-\right. \\
\left.-\varrho v^{\alpha}\left[w_{\alpha}-u_{\alpha}\left(x, t_{0}\right)\right]\right\} d V .
\end{gathered}
$$

Здесь через $\tau^{i k}, \sigma^{i k}, \lambda^{i}, \vartheta^{i}, v^{i}$ и $\mu^{i}$ обозначены множители Лагранжа.

Отметим, что в функционале (2.1) независимыми варьируемыми функциями являются перемещения $u_{i}$, компоненты кинетических тензоров $\varepsilon_{i k}, e_{i k}, v_{i}$ и множители Лагранжа - $\tau^{i k} ; \sigma^{i k}, \lambda^{i}, \vartheta^{i}, v^{i}$ и $\mu^{i}$.

Необходимые условия стационарности функционала (2.1) следующие: 
1) уравнение равновесия

$$
\nabla_{a} \sigma^{a i}+X^{i}-\varrho \frac{\partial \lambda^{i}}{\partial t}=0
$$

2) соотношения между кинетическими тензорами и перемещениями $(1.9),(1.10)$ и $(1.12)$;

3) соотношения упругости

$$
\begin{gathered}
\tau^{i k}-\varrho \frac{\partial F}{\partial \varepsilon_{i k}}=0 \\
\sigma^{i k}-\left(\tau^{i k}+g^{k \alpha} e_{\beta \alpha} \tau^{i \beta}\right)=0 \\
v^{i}-\lambda^{i}=0
\end{gathered}
$$

4) граничные условия

$$
\sigma^{\alpha i} n_{\alpha}-P^{i}=0
$$

на части $S_{1}$ поверхности $S$;

5) граничные условия

$$
\begin{gathered}
\sigma^{2 i} n_{\alpha}-\theta^{i}=0 \\
u_{i}^{\prime}-u_{i}=0
\end{gathered}
$$

на части $S_{2}$ поверхности $S$ и

6) условия (1.15), (1.16) и

$$
\begin{array}{r}
\lambda^{i}\left(x, t_{1}\right)-\mu^{i}=0, \\
\lambda^{i}\left(x, t_{0}\right)-v^{i}=0 .
\end{array}
$$

Из приведенных соотношений видно, что тензоры $\tau^{i k}$ и $\sigma^{i k}$ надо рассматривать как тензоры напряжения, соответствующие кинетическим тензорам $\varepsilon_{i k}$ и $e_{i k}$.

\section{3. Вариационная задача Кастильяно}

Чтобы получить преобразования, дающие вариационную задачу Кастильяно, надо потребовать, чтобы варьируемые функции в общей вариационной задаче удовлетворяли всем условиям-стационарности этой задачи, кроме чисто геометрических. Такими геометрическими условиями являются соотношения (1.9) и (1.12), геометрические граничные условия (2.8) и условия (1.15) и (1.16). Итак, считаем, что варьируемые функции удовлетворяют предварительно следующим условиям стационарности функционала $(2.1)$ : $(2.2),(1.10),(2.3)-$ (2.7) и $(2.9),(2.10)$. Теперь, в случае физически линейной задачи, т. е. при (1.11), функционал (2.1) упрощается к виду

$$
\begin{aligned}
\int_{t_{0}}^{t_{1}} \int_{V}\left(\sigma^{\alpha \beta} e_{\alpha \beta}\right. & \left.+\frac{1}{2} \tau^{\alpha \beta} \varepsilon_{\alpha \beta}-\frac{1}{2} \varrho v^{\alpha} v_{\alpha}\right) d V d t-\int_{t_{0}}^{t_{1}} \int_{S_{2}} \sigma^{\alpha \beta} n_{\alpha} l l_{\beta}^{\prime} d S d t+ \\
& +\int_{V}\left[\varrho v^{\alpha}\left(x, t_{1}\right) w_{\alpha}^{\prime}-\varrho v^{\alpha}\left(x, t_{0}\right) w_{\alpha}\right] d V
\end{aligned}
$$


причем функциональные аргументы этого функционала должны удовлетворять соотношениям

$$
\begin{gathered}
\tau^{i k}=E^{i k \alpha \beta} \varepsilon_{\alpha \beta}, \\
\nabla_{\alpha} \sigma^{\alpha i}+X^{i}-\frac{\partial v^{i}}{\partial t}=0, \\
\sigma^{i k}=\tau^{i \alpha}\left(\delta_{\alpha^{*}}{ }^{k}+e_{\alpha^{*}}{ }^{k}\right),
\end{gathered}
$$

условию (1.10) и граничным условиям (2.6).

Следовательно, вариационная задача Кастильяно в динамике нелинейной теории упругости гласит:

найти стационарное значение функционала (3.1) при предварительных условиях (3.2)-(3.4), (1.10) и (2.6).

Рассмотрим подробнее полученную задачу. Во-первых, сформулируем вариационную задачу Кастильяно через варьируемые функшии $e_{i k}$ и $v_{i}$. Для этого выразим $\tau^{i k}, \sigma^{i k}$ и $\varepsilon_{i k}$ через $e_{i k}$ при помоши соотношений $(3.2),(3.4),(1.10)$ и подставим в функционал (3.1). При варьировании полученного функционала выражения из $e_{i k}$

$$
\sigma^{i k}=\frac{1}{2} E^{i \alpha \beta \gamma}\left(\delta_{\alpha}^{* k}+e_{\dot{\alpha} \cdot k}^{*}\right)\left(e_{\beta \gamma}+e_{\gamma \beta}+e_{\beta}^{\dot{\gamma}} \cdot e_{\gamma \hat{\gamma}}\right)
$$

должны удовлетворять уравнениям равновесия (3.3) и статическим траничным условиям (2.6).

Для удобного практического применения этой вариационной задачи нужно $e_{i k}$ выразить через функции напряжений так, чтобы $e_{i k}$ удовлетворяли тождественно уравнениям равновесия. Легко найти выражения через функции напряжений для $\sigma^{i k}$ и $v^{i}$. Но далее, при выражении $e_{i k}$ через эти функции, мы встретимся с большими алгебраическими трудностями, так как мы должны решить систему (3.5) из девяти кубических уравнений.

Во-вторых, сформулируем вариационную задачу Кастильяно через варьируемые функции $\tau^{i k}$ и $v^{i}$. Из (3.4) получаем, что

$$
e_{i k}=\frac{3 c_{i b \xi} c_{\alpha \beta \gamma}\left(\sigma_{* k}^{\alpha}-\tau_{* k}^{\alpha}\right) \tau^{\beta \hat{\sigma}} \tau^{\gamma \xi}}{c_{\rho \omega \alpha} c_{v \mu \varepsilon} \tau^{\rho \nu} \tau^{\omega \mu \mu} \tau^{\chi \beta}} .
$$

Если считать, что $\sigma^{i k}$ и $v^{i}$ выражены через функции напряжений, $\tau^{i k}$ удовлетворяют при помощи (1.10) и (3.6) полученным уравнениям упругости и если в функционале (3.1) сделать соответственные подстановки, получаем искомую вариационную задачу. Трудности, возникающие при практическом использовании этой вариационной задати, также носят алгебраический характер.

В заключение отметим, что из сформулированной вариационной задачи динамики получается вариационная задача статики нелинейной теории упругости, если взять $v^{i}=0$ и считать использованные тензоры не зависящими от времени. 


\title{
ЛИТЕ Р А Т У Р А
}

1. A. Phillips, Variational principles in the theory of finite plastic deformations, Quart. Appl. Math., Vol. 7, No. 1, 1949

2. K. 3. Г а ли м о В, К вариационным принципам нелинейной теории упругости, Уч. зап. Казанск. ун-та, т. 113, кн. 10, 1953.

3. E. Ko p p e, Die Ableitung der Minimalprinzipien der nichtlinearen Elastizitätstheorie mittels kanonischer Transformation, Nachr. Akad. Wiss. Göttingen. Math. phys. K1. II a, Nr. 12, 1956.

4. R. F. B a l d a c c i, Sopra un principio variazionale nella teoria degli spostamenti elastici non infinitesimi, Atti Accad. Naz. Lincei. Rend. Cl. Sci. fis., mat e natur., vol. 23, № $5,1957$.

5. I. Н. Слезінгер, Принцип Кастільяно в нелінійній теоріі пружності, Прикл. механіка, т. V, в. 1, 1959.

6. E. Re is s ner, Note on the method of complementary energy, Journ. Math. Phys., vol. 27, No. 2, 1948.

7. H, C. Hu, On two variational principles for the natural vibrations of elastic bodies. Chinese J. Mech., vol. I, No. 2, 1957.

8. Р. Кураит и Д. Гильберт, Методы математической физики, т. I, гл. IV, $\S 9,1951$.

Таллинский политехнический институт
Поступилаи в редакцию

28. III 1960

\section{MITTELINEAARSE ELASTSUSTEOORIA DUNAAMIKA CASTIGLIANO VARIATSIOONULESANDEST}

\author{
L. Ainola,
}

\author{
füüsikalis-matemaatiliste teaduste kandidaat
}

\section{Resümee}

Uldise variatsioonülesannete teisendamise meetodiga tuletatakse Hamiltoni variatsioonülesandest geomeetriliselt mittelineaarse elastsusteooria dünaamika jaoks Castigliano variatsioonülesanne.

Tallinna Polütehniline Instituut
Saabus toimetusse

28. III 1960

\section{ON CASTIGLIANO'S VARIATIONAL PRINCIPLE IN THE THEORY OF DYNAMICS OF FINITE ELASTIC DEFORMATIONS}

\section{Ainola}

\section{Summary}

By means of the general method of transformation of variational equations Castigliano's variational principle for the theory of dynamics of finite elastic deformations is deduced from the Hamiltonian principle. 